\title{
Computerunterstütztes Entwerfen, Gestalten und Nachrechnen von Stirnradgetrieben in der Ingenieurausbildung
}

\author{
Prof. Dipl.-Ing. Karin Jannasch
}

\section{Vorwort}

Die Berechnung und Konstruktion eines Getriebes gehört zu den Standardaufgaben in der Ausbildung von Maschinenbauingenieuren. Kaum eine andere Problematik zeigt so deutlich bei Berechnung und Gestaltung die Abhängigkeiten der einzelnen Komponenten voneinander. Diese Arbeit für den Studierenden überschaubar zu halten und ihm dennoch die Möglichkeit zu zeigen, verschiedene Dimensionierungs- und Gestaltungsvarianten zu untersuchen, ist ein Hauptanliegen für den Lehrenden. So wurde an der damaligen Ingenieurschule fuir Maschinenbau Wildau bereits 1980 begonnen, mit einfachen Tischrechnern aufwendige Variantenberechnungen für einzelne Maschinenteile zeitsparend durchzuführen. Mit dem heutigem Stand des CAD-Labors der Technischen Fachhochschule sowie der verfuigbaren Anwendersoftware bieten sich neue Möglichkeiten der durchgängigen Bearbeitung eines Getriebes am Computer. Unter Nutzung dieser Möglichkeiten entstand in den letzten zwei Jahren das Programm WCAD, das sowohl eigene Berechnungsmodule enthält, als auch bekannte Programme (AutoCAD, ME DESIGN) einbezieht.

\section{Problemstellung und Erarbeitung der Anforderungen}

An der Ingenieurschule für Maschinenbau Wildau wurden bis 1989 von Studenten verschiedene Computerprogramme zur Berechnung von Getriebewellen erarbeitet, die jedoch den heutigen Anforderungen nicht standhalten. Sie sind größtenteils in BASIC und für Kleincomputer programmiert, die nur über geringe Speicherkapazitäten und kleine Rechengeschwindigkeiten verfügen. Da diese Programme zur neuen PC-Generation nicht mehr kompatibel sind, wurde mit der Modernisierung des CAD-Labors an der Technischen Fachhochschule Wildau eine völlige Neukonzipierung der Programme unter Einbindung vorhandener Nachrechnungs- und Grafikprogramme erforderlich.

Für den Computereinsatz im Konstruktionsprozeß stehen heute vielfältige Programme zur Verfuigung. Mit ihnen ist die Bearbeitung von einzelnen Teilaufgaben, wie z. B. Zeichnungserstellung oder Nachweisrechnung einzelner Maschinenelemente, in guter Qualität möglich. Durch die Zeitersparnis arbeitet der Konstrukteur effizienter. Weiterhin sind umfangreiche Variantenuntersuchungen möglich.

Im Gegensatz zu der Nachrechnung von Maschinenbauteilen, die meistens nach vorhandenen Berechnungs- algorithmen und einer konkreten Zuordnung von Werten erfolgt, ist in der Entwurfsphase die Kreativität und Erfahrung des Ingenieurs besonders gefragt. So ist im Entwurfsstadium oftmals eine exakte Nachweisrechnung noch nicht möglich, da wichtige Abmessungen fehlen ( $\mathrm{z}$. B. Lagerabstand einer Getriebewelle), und erst bei der Nachweisrechnung können Aussagen über die Realisierbarkeit getroffen werden. Das ist ein wesentlicher Grund, warum in dieser Entwicklungsphase kaum Dimensionierungsprogramme zur Verfügung stehen. In der Industrie, wo der Konstrukteur von seinen Erfahrungen profitieren und in der Regel auf vorhandene Konstruktionen zurückgreifen kann, ist hierfür auch keine ausgesprochene Notwendigkeit vorhanden. Bei den Studenten fehlt aber oftmals noch das Gefühl für das komplexe Zusammenspiel der verschiedenen Einflußgrößen auf eine Konstruktion und für die richtigen Größenordnungen der anzunehmenden Werte, so daß die Ergebnisse oft weit entfernt vom Optimum liegen.

Ein weiterer Nachteil marktüblicher Grafik- und Berechnungsprogramme ist die fehlende Vernetzung untereinander. Damit ist keine Datenübergabe zwischen den einzelnen Berechnungsmodulen möglich, was eine mehrfache Dateneingabe notwendig macht. Das ist zeitintensiv und schließt auch Eingabefehler nicht aus. Die Kopplung zwischen Berechnung und Grafik wird inzwischen von einigen Programmen ermöglicht (GENIUS für Auto$C A D, A C A D M E$ u. a. m.). Dabei werden aber immer nur einzelne Elemente, wie z. B. Paßfeder, Schraube oder Wälzlager, ausgewählt und grafisch angezeigt.

Wenn eine Entwicklungsaufgabe algorithmierbar ist, muß es auch möglich sein, diese durchgängig am Computer zu bearbeiten. Als Beispiel für eine durchgängige computergestützte Konstruktionslösung wurde hierbei die Entwicklung eines zweistufigen Stirnradgetriebes gewählt. Diese Aufgabe hat in der Ausbildung von Maschinenbaustudenten einen festen Platz. Es werden Inhalte des Lehrgebietes Maschinenelemente angewendet und gefestigt, und zugleich muß vom Bearbeiter eine hohe Komplexität bei Berechnung und Gestaltung beachtet werden.

An das neuzuentwickelnde Programm sind folgende Anforderungen zu stellen:

\section{Allgemeine Anforderungen:}

- durchgängige Bearbeitung am PC vom Entwurf bis zur Dokumentation,

- theoretische Grundlagen entsprechend Lehrplan und Fachliteratur,

- Datenübergabe zwischen den einzelnen Programmmodulen, 
- Datenmenge übersichtlich strukturiert,

- Datenmenge beliebig ausbaubar,

- Benutzerfreundlichkeit,

- uibersichtliche Bildschirmgestaltung,

- Vermeidung vom Eingabefehlern und Fehlbedienung,

- Zuverlässigkeit,

- Protokolldruck,

- Anwenderdokumentation,

- Programmdokumentation,

- Schnittstellen zu Grafik und Nachrechnungsprogrammen,

- einfache Installation und Konfiguration,

- Verwaltung der Dateien unabhängig vom Programm.

Anforderungen aus der Sicht der Lehre:

- Hilfestellung bei Auswahl von Kennwerten und konstruktiven Entscheidungen,

- Überprüfung hinsichtlich konstruktiver Realisierbarkeit,

- Speicherung der Daten unter Seminargruppe, Name und Variante,

- theoretische Grundlagen entsprechend Lehrplan und Studienliteratur.

Anforderungen entsprechend der Konstruktionsaufgabe:

- Bearbeitung von maximal 2-stufigen Stirnradgetrieben mit gehärteter Gerad- oder Schrägverzahnung,

- Leistung $5 \ldots 40 \mathrm{~kW}$ bei Drehzahlen bis max. $1500 \mathrm{~min}-1$,

- Übersetzung 8 bis 40 ,

- Wälzlagerung: Rillenkugellager, Zylinderrollenlager und Kegelrollenlager,

- Paßfeder, Keilwelle oder Querpreßverbindung,

- Wellen horizontal in einer Ebene, Stufen nacheinander angeordnet (kein Koaxialgetriebe),

- Einsatz von genormten Maschinenteilen und Formelementen.

Anforderungen entsprechend der verfügbaren Hardware:

- Anwendung am PC, 286 aufwärts mit mind. IMB RAM,

- EGANGA-Grafik wahlweise,

- Ansteuerung aller Drucker,

- Speicherung der Daten im Netz.

\section{Theoretische Grundlagen der Entwurfs- berechnung von Stirnradgetrieben}

\subsection{Berechnungsverfahren für die Dimensionierung der Getriebeelemente}

\subsubsection{Dimensionierung der Zahnräder}

Bei der Entwurfsberechnung von Zahnradgetrieben sind am Anfang mehrere voneinander abhängige Größen unbekannt. Das macht eine iterative Lösung notwendig, wobei eine Vorwahl von Hauptabmessungen auf der Grundlage von Erfahrungswerten erfolgen muß. Um mit möglichst wenig Iterationsschritten eine zufriedenstellende Lösung zu erhalten, werden in der Literatur verschiedene Reihenfolgen in der Berechnung und Empfehlungen für die Wahl der jeweiligen Ausgangsgrößen angegeben. Da die Verzahnungsgeometrie und damit die
Abhängigkeiten der einzelnen Größen voneinander festgelegt sind, sind die Unterschiede im Berechnungsalgorithmus der Verzahnung aber nicht wesentlich. Im folgenden sollen die bekanntesten Dimensionierungsmöglichkeiten kurz eingeschätzt werden.

\subsubsection{Entwurfsberechnung nach Zirpke [4]}

Beim Entwurf nach Zirpke wird zu Beginn der Verzahnungsberechnung mit einer Überschlagsformel der erforderliche Modul der Verzahnung ermittelt. Dabei wird unterschieden, ob möglicherweise die Zahnflanken verschleißen werden oder eher der Zahn ausbrechen kann.

Modul für ungehärtete Räder (Flankenpressung ausschlaggebend)

$$
\mathbf{m} \approx \frac{10 \cos \beta}{\mathbf{z}_{1}} \sqrt[3]{\frac{\mathbf{y}_{\mathrm{S}} \mathrm{TK}_{\mathrm{A}}}{\mathrm{b}} \sigma_{\mathrm{H}_{\mathrm{zul}}}{ }^{2} \frac{\mathrm{u}+\mathbf{1}}{\mathbf{u}}}
$$

ys Fakțor für Werkstoffpaarung nach Anm. 4

Modul fuir gehärtete Räder (Biegung am Zahnfuß ausschlaggebend):

$$
m \approx \sqrt[3]{\frac{4 T K_{A} \cos ^{2} \beta}{z_{1}{ }^{2} \frac{b}{d} \sigma_{F_{m i}}}}
$$

Zahnräder, die nach Gl. 2-2 entworfen wurden, weisen bei der Nachrechnung ausreichende Sicherheiten gegen Bruch aus. Die Flankentragfähigkeit wird dagegen mit normalen Geometriewerten nicht immer erreicht, spezielle Zahnkorrekturen, wie z. B. Balligkeit oder höhere Verzahnungsqualität, werden nötig (s. Bild 2-1).

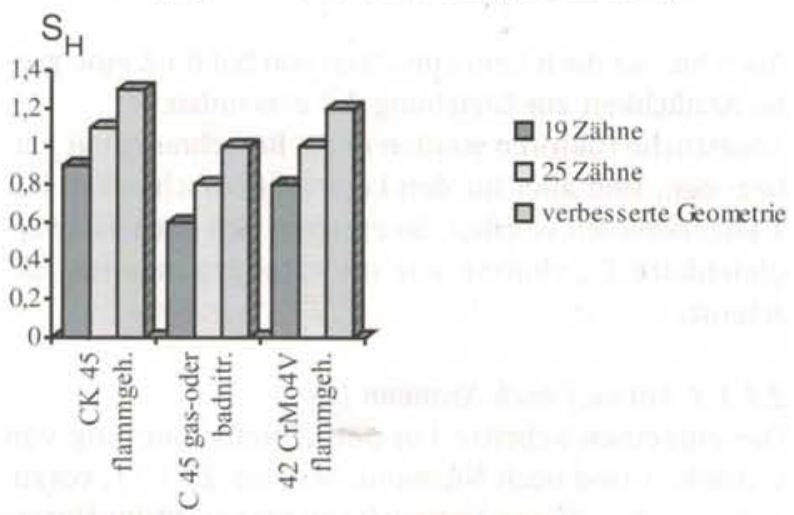

Bild 2-1 erreichte Sicherheiten bei der Nachrechnung

Eine Erklärung dafür ist die gegenüber $[2]$ relativ einfache Nachrechnung der Pressung. So wurden in den letzten Jahren eine Reihe von Faktoren zur besseren Beurteilung der Zahnflankentragfähigkeit eingefuihrt, die bei der Entwurfsberechnung nach Zirpke noch nicht berüicksichtigt sind. Ein anderer Grund liegt darin, daß bei bestimmten Werkstoffen und Wärmebehandlungsverfahren die erreichbare Härte die Grübchenfestigkeit beeinflußt, so daß das Verhältnis $s_{H}$ zu $s_{F}$ besonders ungünstig wird. Beim Entwurf gehärteter Verzahnungen ist deshalb zu beachten:

- Für einfache (billige) Verzahnungsgeometrie und bei kleinen Ritzelzähnezahlen sollte großzuigig aufgerundet werden. 
- Wenn mit geringen Härtewerten gerechnet werden muß, ist ein weiterer Entwurf über die zulässige Flankenpressung ratsam.

- Verbesserungen an der Verzahnungsgeometrie sind möglich und für leistungsfähige Getriebe unbedingt zu empfehlen.

\subsubsection{Entwurf nach Köhler/Rögnitz [8]}

Maßgeblich für den Entwurf nach Köhler/Rögnitz ist wie bei Zirpke der erforderliche Modul der Verzahnung. Für gehärtete Verzahnungen wird der Modul unter Beachtung der Zahnfuß-Tragfähigkeit nach folgender Gleichung empfohlen:

$$
\mathbf{m} \geq \sqrt[3]{\frac{2 \mathrm{~T} \cos \beta}{\mathrm{z}_{1} \lambda \sigma_{\mathrm{F}_{\mathrm{zal}}}} \mathbf{Y}_{\mathrm{F}} \mathbf{Y}_{\varepsilon} \mathbf{Y}_{\beta} \mathbf{K}_{\mathrm{F} \alpha}}
$$

Setzt man für die Faktoren $\mathrm{Y}_{\mathrm{F}}=2,2$

$$
\begin{aligned}
& \mathrm{Y}_{\mathrm{e}}=1 \\
& \mathrm{~K}_{\mathrm{Fa}}=1,
\end{aligned}
$$

und ersetzt

$$
\lambda^{\mathrm{a}}=\mathrm{b} / \mathrm{m}=\mathrm{b} \mathrm{z}_{1} /(\mathrm{d} \cos \beta) \text {, }
$$

erhält man ähnliche Ergebnisse wie im vorangegangenen Abschnitt.

Für ungehärtete Räder ist die Modulberechnung unter Beachtung der Flankentragfähigkeit maßgebend und wird nach der folgenden Gleichung durchgeführt.

$$
\mathbf{m} \geq \sqrt[3]{\frac{\mathbf{u}+1}{\mathbf{u}} \frac{2 \mathrm{~T} \cos ^{2} \beta}{\mathbf{z}_{1}{ }^{2} \lambda \sigma_{\mathrm{H}_{\mathrm{zut}}}{ }^{2}} \mathbf{K}_{\mathrm{H} \alpha} \mathrm{Z}_{\mathrm{M}}{ }^{2} \mathrm{Z}_{\mathrm{H}}{ }^{2} \mathrm{Z}_{\varepsilon}{ }^{2}}
$$

$$
\begin{aligned}
& \mathrm{KHa}=1 \\
& \mathrm{Z}_{\mathrm{M}}=270 \sqrt{\mathrm{N} / \mathrm{mm}^{2}} \text { (für St/St) }
\end{aligned}
$$

Auch hier ist nach dem Einsetzen von b/d für $\lambda$ eine große Ähnlichkeit zur Gleichung 2-2 erkennbar.

Zusätzliche Faktoren werden in die Berechnung mit einbezogen, sind aber für den Entwurf überschläglich mit 1 angenommen worden. So ergeben sich auch hier vergleichbare Ergebnisse wie im vorangegangenen $\mathrm{Ab}$ schnitt.

\subsubsection{Entwurf nach Niemann [2]}

Die einzelnen Schritte bei der Dimensionierung von Getrieben sind nach Niemann, Schema 22.1.11, vorzunehmen. Bei dieser Entwurfsvariante wird im Unterschied zu den vorherigen Beispielen zuerst der Teilkreisdurchmesser und mit diesem der Modul bestimmt. Die Entwurfsgleichung für den Teilkreisdurchmesser wird aus der Gleichung für die Nachrechnung der Flankenpressung durch Umstellung nach dem Teilkreisdurchmesser hergeleitet. Damit ergibt sich eine ähnliche Gleichung wie bei $[4]$ und [8] für ungehärtete Verzahnungen.

$$
\begin{aligned}
& d_{1}=\sqrt[3]{\frac{2000 T}{K *\left(\frac{b}{d_{1}}\right)} \frac{u+1}{u}} \\
& K^{*}=\frac{F_{1}}{d_{1} b} \frac{u+1}{u}
\end{aligned}
$$

Im Faktor $\mathrm{K}^{\circ}$ wurden die verschiedenen Einflußfaktoren von Geometrie und Werkstoff zusammengefaßt. Damit sind die einzelnen Einflüsse nicht mehr klar abgrenzbar. So ist z. B. keine Aussage über Festigkeitswerte des Werkstoffes möglich. Der Faktor $\mathrm{K}^{\circ}$ wird entsprechend des geplanten Einsatzes des Getriebes nach [2], Tafel $22.1 / 2$, ausgewählt und kann zwischen 1,3 bis 24 liegen. Für immer wiederkehrende praktische Anwendungen ist diese Verfahrensweise einfach und vorteilhaft, da hier konkrete Erfahrungswerte für $\mathrm{K}^{\circ}$ vorliegen. Für den Studenten allerdings ist es schwer, nach der Wahl von Werkstoff und Verzahnungsqualität den richtigen Wert von $\mathrm{K}^{\circ}$ zu bestimmen. Die zur Verfügung stehenden Tabellen sind dafür kaum geeignet. So ist eine iterative Nachprüfung des Faktors notwendig.

\subsubsection{Empfehlungen für den Entwurf von Verzahnungen} Für die konstruktive Auslegung der Verzahnung ist in Bild 2-2 ein Berechnungsablauf dargestellt, der sich im wesentlichen auf den Entwurf des Moduls nach Zirpke bezieht. Hinweise zur Wahl von Kennwerten und konstruktiven Entscheidungen basieren auf allen Literaturhinweisen der vorangegangenen Abschnitte, sowie auf eigenen Erfahrungen bei Entwurf und Nachrechnung von Getrieben.

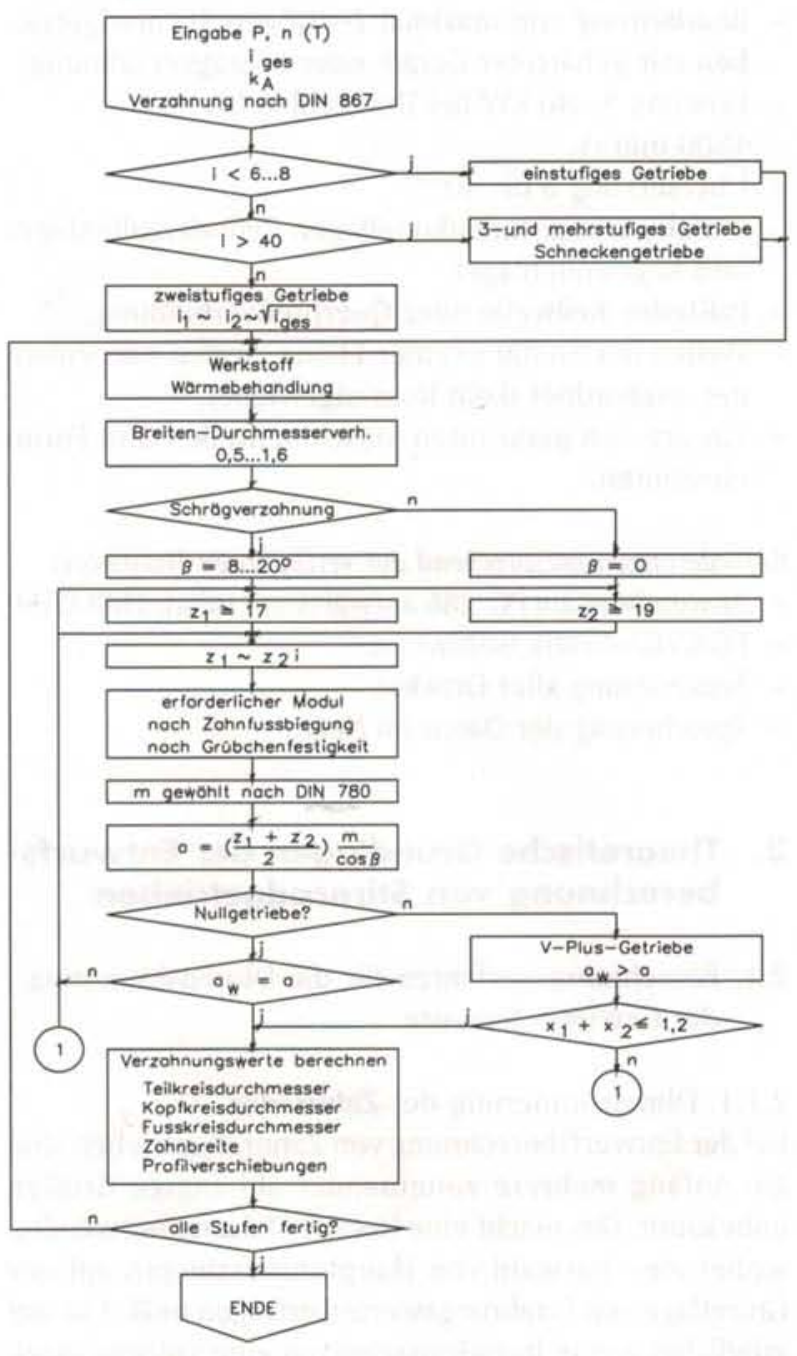

Bild 2-2: Ablaufplan zum Entwurf von Verzahnungen

Erläuterungen zum Ablaufplan

Dieser Ablaufplan zeigt einige Möglichkeiten auf, wie 
beim Entwerfen von Zahnradstufen vorgegangen werden kann. In der Regel werden als Eingangsgrößen die zu übertragende Leistung, Eingangsdrehzahl sowie die geforderte Gesamtiibersetzung vorgegeben. Entsprechend dem geplanten Einsatz für das Getriebe sind der Anwendungsfaktor und die geforderte Sicherheit zu wählen.

Üblich sind nach [2] für Gesamtübersetzungen bis 6 (evtl. 8) einstufige, bis 35 (evtl. 45) zweistufige und bis 150 (evtl. 200) dreistufige Getriebe. Für die Aufteilung sind in der Literatur (z. B. [2], [6]) verschiedene Empfehlungen enthalten. Hiernach kann für den ersten Überschlag für beide Stufen die gleiche Teilübersetzung gewählt werden, aber auch unterschiedliche Aufteilungen sind denkbar. Eine große Übersetzung in der ersten Stufe ist oft erwünscht, da hier die Gesamtgröße noch nicht entscheidend beeinflußt wird. Auch ist ein größerer Achsabstand für die konstruktive Gestaltung der Lagerstellen von Vorteil. Es kann aber auch möglich sein, daß bei einer zu großen Übersetzung in der ersten Stufe das Eingangsdrehmoment für die zweite Stufe so hoch wird, daß ein größerer Modul in der zweiten Stufe erforderlich wird, der das gesamte Getriebe entscheidend vergrößert. Genaue Aussagen sind hier nur nach dem Vergleich verschiedener Varianten möglich. Ganzzahlige Teilübersetzungen sind möglichst zu vermeiden, da es sonst zu ungleichem Einlaufen der Zahnräder kommt. Bei der Auswahl der Werkstoffe sollte beim ersten Entwurf noch nicht der Werkstoff mit der höchsten Festigkeit und Flankenhärte ausgewählt werden. In der zweiten Stufe kann der Werkstoff etwas hochwertiger sein, da hier größere Abmessungen zu erwarten sind. $\mathrm{Zu}$ beachten ist auch der notwendige Härtesprung zwischen Rad und Ritzel!

Für die Wahl des Breiten-Durchmesserverhältnisses sind Werte von 0,6 bis 0,8 empfehlenswert. Breitere Räder müssen genauer gefertigt werden, die Lagerung der Wellen ist aufwendiger. Die Räder werden dann meist so klein, daß der Fußkreisdurchmesser kleiner wird als der notwendige Wellendurchmesser.

Schrägverzahnungen bringen den Vorteil des ruhigeren Laufes, der größeren Überdeckung und der kleineren Abmessungen. Allerdings ist die Fertigung aufwendiger, und es entstehen an der Verzahnung zusätzliche axiale Kräfte. Aus diesem Grund sollten die Schrägungswinkel nicht zu groß gewählt und zwischen den Stufen darauf geachtet werden, daß sich die Axialkräfte möglichst gegenseitig aufheben. Durch Variieren des Schrägungswinkels kann der rechnerische Achsabstand an den Nullachsabstand angepaßt werden.

Die Zähnezahl des Ritzels ist nicht zu klein zu wählen. Die Grenzzähnezahl 14 bzw. 11 für Schrägverzahnungen ist bereits problematisch. Solche Getriebe sind nur anhand von Nachrechnungen auszuwählen. Für den Entwurf sollte die Ritzelzähnezahl 19 bzw. 17 bei Schrägverzahnung nicht unterschritten werden. Wird eine hohe Grübchentragfähigkeit gewünscht, sollte die Ritzelzähnezahl bei etwa 25 bis 30 liegen. Größere Ritzelzähnezahlen verteuern allerdings auch die Fertigung! Die Radzähnezahl sollte sich in einem Bereich befinden, der die gewählte Teilübersetzung in etwa realisieren kann. Bei der letzten Stufe ist die Kontrolle der zulässi- gen Abweichung von der Gesamtübersetzung notwendig. Die Zähnezahl des Rades sollte kein Vielfaches der Ritzelzähnezahl sein, damit ein gleichmäßigerer Einlauf des Radpaaren möglich wird.

V-Minus-Getriebe sollten nicht zum Einsatz kommen, da durch die Schwächung am Zahngrund die Zahnfußwechselfestigkeit ungünstig beeinflußt wird. Dagegen sind Räder mit positiven Profilverschiebungswerten zu empfehlen. Bei V-Plus-Getrieben vergrößert sich durch die Verschiebung des Profils der Zahnflanken die Dicke des Zahnes. Die Biegewechselfestigkeit erhöht sich. Nullgetriebe haben die einfachste Verzahnungsgeometrie, was aber bei der Herstellung auf modernen Verzahnungsautomaten kaum noch einen Einfluß hat.

Ist die Verzahnung überschläglich so dimensioniert, daß die Hauptanforderungen an das Getriebe erfüllt sind, sollten zunächst die übrigen Getriebebauteile wie Wellen, Mitnehmerverbindungen und Lager dimensioniert werden. Erst hier zeigt sich nach Skizzierung des Gesamtgetriebes, ob das Getriebe so realisierbar ist oder eventuell die Verzahnung neu entworfen werden muß. Damit ist der Grobentwurf beendet. Ob das Getriebe so ausgefuihrt werden kann, zeigt sich aber erst nach genauer Berechnung der Verzahnungsgeometrie sowie der Tragfähigkeit der Zähne.

\subsubsection{Dimensionierung von Wellen}

Die Bestimmung des Wellendurchmessers ist in einem frühen Entwurfstadium nötig, da sich andere Maschinenteile auf diesen Wert beziehen. Zu diesem Zeitpunkt kann noch keine Aussage über die genaue Belastung der Welle gemacht werden, da aufgrund fehlender Stützweiten der Biegeanteil nicht bekannt ist. So ist die Grobdimensionierung der Welle vorerst nur über das Drehmoment möglich.

Nach [2], [3] und [8] kann der Wellenquerschnitt wie folgt ermittelt werden:

$$
d_{\text {erf }}=\sqrt[3]{\frac{16 T}{\pi \tau_{\text {tüb }}}} \approx 1,72 \sqrt[3]{\frac{T}{\tau_{\text {tüb }}}}
$$

Nach [3] wird für die überschlägliche Torsionsspannung ttuib $=\left(\begin{array}{lll}12 & \ldots & 30\end{array} 5\right)$ MPa vorgeschlagen, der kleinere Wert für große Stützweiten und hohen Biegeanteil, der größere Wert für geringe Stützweiten und geringen Biegeanteil. Für höherfeste Werkstoffe ist ebenfalls ein größerer Wert einzusetzen. Nach [2] und [8] werden für die überschlägliche Torsionsspannung folgende Werte empfohlen:

$$
\begin{aligned}
& \text { [2] } \left.\tau_{\text {tüb }}=(14 \ldots 18) \mathrm{MPa} \text { (für St } 50\right) \text { oder } \\
& \tau_{\text {tüb }}=\tau_{\text {tsch }} /(12 \ldots 14) \\
& {[8] \tau_{\text {tüb }} 15 \mathrm{MPa} .}
\end{aligned}
$$

Nach [6] kann der Wellendurchmesser bei unbekannter Biegung auch nach folgender Gleichung bestimmt werden.

$$
d_{\text {erf }} \geq \sqrt[3]{\frac{32 M_{v}}{\pi \sigma_{b z u l}}} \approx(3,5 \ldots 4,5) \sqrt[3]{\frac{T}{\sigma_{b w}}}
$$

Hierbei wurde die zulässige Biegespannung ersetzt durch $\sigma_{\text {bzul. }}=\sigma_{b w} /(3 \ldots 4)$ und das Vergleichsmoment 
aus der Vergleichspannung nach der GestaltänderungsEnergiehypothese abgeleitet. Das unbekannte Biegungmo-ment im Vergleichsmoment wird bei kleinen Stützweiten in gleicher Höhe wie das Torsionsmoment angenommen (kleinerer Wert in Gl. 2-10) und bei großen Stiitzweiten doppelt so hoch (größerer Wert in Gl. 2-10). Der Werkstoffeinfluß bei der Dimensionierung der Welle ist durch die Biegewechselfestigkeit $\sigma_{\mathrm{bw}}$ berïcksichtigt. Beide Gleichungen ermöglichen einen groben Entwurf des Wellendurchmessers, wobei nach Gleichung 2-9 die Möglichkeit gegeben ist, Erfahrungswerte entsprechend bereits ausgefuihrten Wellen besser einfließen zu lassen. Bei der Verwendung von Gleichung 2-10 in der Lehre ist $\mathrm{zu}$ bedenken, daß in der Berechnungsgleichung ein grundsätzlicher Fehler enthalten ist, indem die Biegespannung aus dem Drehmoment ermittelt wird! Die eigentlichen Überlegungen bei der Herleitung der Formel sind bei der Anwendung nicht mehr klar erkennbar. Da die Welle nicht isoliert von den angrenzenden Bauteilen wie Kupplung, Lager oder Rädern gesehen werden darf, ist auch der Wellendurchmesser diesen Maschinenteilen gegebenenfalls anzupassen. Dazu werden die entsprechenden Bauteile ausgewählt und konstruktiv auf die Welle abgestimmt. Dabei kann es durchaus nötig sein, den überschläglich ermittelten Wellendurchmesser zu vergrößern.

Sind die Belastungsgrößen der Welle bekannt, ist ein genauerer Entwurf möglich. Dieser erfolgt nach [3] über die Vergleichsausschlagspannung, wobei wechselnde Biegung und schwellende oder statische Torsion angenommen wird. Entsprechend der zu erwartenden Kerbwirkung kann in Abhängigkeit der Zugfestigkeit eine überschlägliche Biegewechselfestigkeit bestimmt werden.

$$
d_{\text {erf }} \geq 2,17 \sqrt[3]{\frac{M_{v}}{\sigma_{\text {baïb }}}}
$$

Der so bestimmte Kerndurchmesser ist entsprechend den querschnittschwächenden Formelementen wie Paßfedernut oder Einstich für Sicherungsring auf den auszuführenden Wellendurchmesser zu vergrößern. Das kann durch Einsatz eines Faktors für Schwächung und Kerbwirkung oder durch einen Zuschlag auf den ermittelten Wellendurchmesser erfolgen.

Ein genauer Nachweis der Dauerfestigkeit ist auf jeden Fall notwendig, da allein mit den Entwurfsgleichungen nicht alle Beanspruchungen der Welle exakt ermittelt werden können.

Neben dem Festigkeitsnachweis der Wellen ist bei Getrieben auch das elastische Verhalten der Wellen zu überprüfen, da eine zu hohe Verformung die Funktion der Welle selbst oder anderer Bauteile erheblich beeinträchtigen kann. Wichtige Überprüfungskriterien sind:

- die Durchbiegung der Wellen unter den Zahnrädern.

$\mathrm{Zu}$ hohe Durchbiegungen vergrößern das Flankenspiel und die Beanspruchung der Zähne, was zu vorzeitigen Ausfall und unerwünschten Geräuschen führen kann. Die Durchbiegung sollte nicht größer als $0,5 \ldots 1 \%$ des Moduls betragen.

- Die Neigung am Zahnrad führt zu ungleichmäßigen Pressungswerten über der Zahnbreite, evtl. auch zu erhöhten Kantenpressungen. Die Neigung wird bei der Nachrechnung der Verzahnung beachtet und durch mögliche Geometriekorrekturen ausgeglichen.

- die Neigung der Welle in den Lagern. Die zulässige Neigung wird durch die Konstruktion der Lager begrenzt.

- Der zulässige Verdrehwinkel soll bei oft wechselnder Drehrichtung oder langen Wellen $0.25^{\circ} / \mathrm{m}$ nicht überschreiten.

Für die exakte Berechnung der Durchbiegung der Wellen ist die endgültige Gestaltung der Welle erforderlich. Im Entwurfstadium kann zunächst von einer glatten Welle ausgegangen werden. Die Stützwirkung der Naben ist vernachlässigbar. Die Verformungen der Welle können für fiktive Balastungen $\mathrm{M}^{\circ}$ und $\mathrm{F}^{\circ}$ wie folgt ermittelt werden $[3]$ :

$$
\begin{array}{ll}
\text { Durchbiegung } & \eta=\frac{1}{\mathbf{E}} \mathbf{M}_{\mathrm{b}} * \\
\text { Neigung } & \eta^{\prime}=\frac{1}{\mathbf{E}} \frac{\mathbf{d} \mathbf{M}_{\mathrm{b}} *(\mathbf{z})}{\mathbf{d z}}=\frac{1}{\mathbf{E}} \mathbf{F}_{\mathrm{Q}} *
\end{array}
$$

Die fiktiven Balastungen sind entsprechend dem vorhandenen Belastungsfall zu bestimmen.

Durchmesser für zulässigen Verdrehwinkel

$$
d_{\text {erf }}=9,24 \sqrt[4]{\frac{T}{\vartheta_{\text {zul }}}}
$$

\subsubsection{Auswahl der Wälzlager}

Die erste wichtige Aufgabe für die Lagerauswahl ist die Feststellung der Belastungsgrößen an den Lagerstellen. Entsprechend den Eingriffsverhältnissen der Zahnräder ist dazu ein räumliches Kräfteschema zu entwickeln und in den einzelnen Ebenen zu berechnen. Wenn Nabenund Lagerbreiten noch nicht bekannt sind, müssen dafür Erfahrungswerte eingesetzt werden. Für einen ersten Entwurf kann $\mathrm{a}=\mathrm{b}=1 / 31$ gelten.

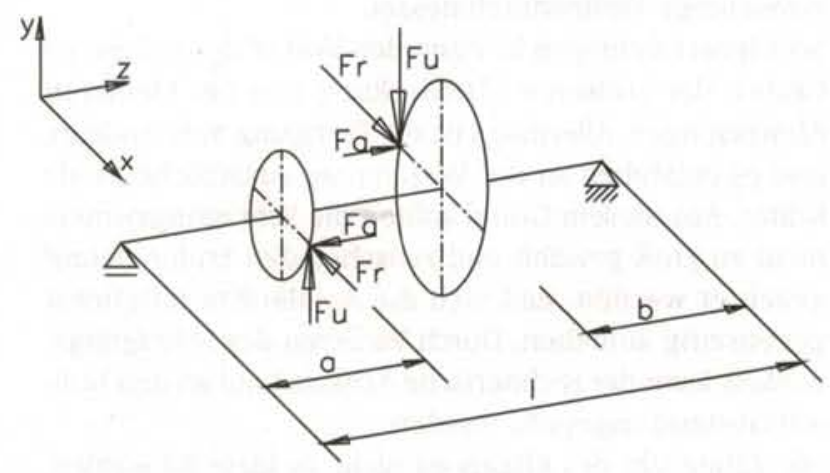

Bild 2-3: Schema zur Ermittlung der Lagerkräfte

$1=2^{*}$ Nabenbreite + konstruktive Abstände + geschätzte Lagerbreite

Als Nabenbreite kann $1,2^{\circ}$ Wellendurchmesser angenommen werden. Die konstruktiv beeinflußbaren Abstände sind geschätzt mit:

Abstand zwischen den Zahnrädern

$5 \mathrm{~mm}$

Abstand Zahnrad - Gehäuse

$10 \mathrm{~mm}$

Gehäuse bis Lagerkante 
Damit beträgt die anzunehmende Stützweite

$$
I=2,4 \cdot d_{w}+35 \mathrm{~mm}+\text { Lagerbreite } .
$$

Die Lagerkräfte werden in den Ebenen $x, z$ und y,z ermittelt und anschließend zu der Axialbelastung im Festlager und den resultierenden Radialbelastungen zusammengefaßt. Entsprechend den Berechnungsvorschriften der Wälzlagerhersteller kann danach ein Lager ausgewählt werden, was die geforderte Lebensdauer mit hoher Wahrscheinlichkeit erreichen wird.

\subsection{Abhängigkeiten bei der Dimensionierung der Getriebeelemente}

\subsubsection{Die Welle als zentrales Bauteil}

Bei der Dimensionierung eines kompletten Getriebes reicht es nicht aus, alle einzelnen Maschinenteile wie in den vorangegangenen Abschnitten beschrieben zu entwerfen. Vielmehr müssen eine Reihe von konstruktiven Abhängigkeiten beachtet werden. Dabei spielt das Bauteil Getriebewelle eine zentrale Rolle, da alle anderen Getriebeelemente darauf montiert werden.

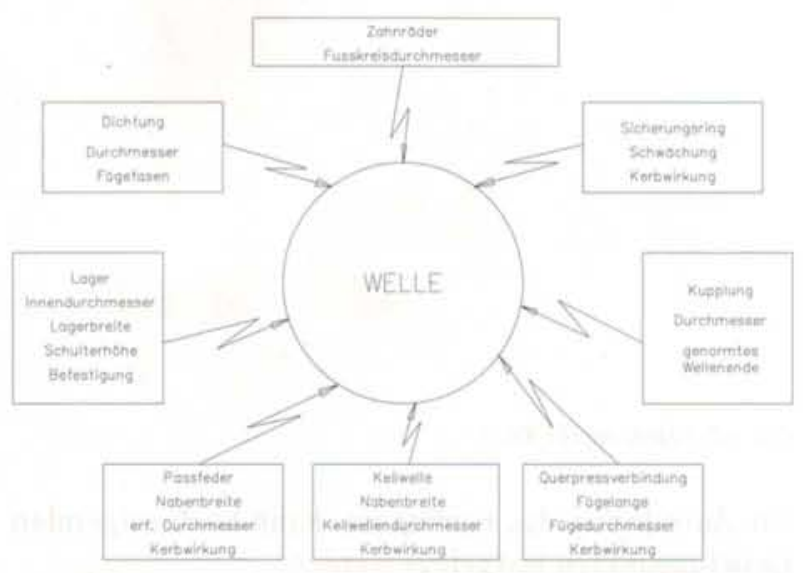

Bild 2-4: Einflïsse auf Dimensionierung und Gestaltung der Getriebewelle

Neben der eigentlichen Beanspruchung der Welle auf Biegung und Verdrehung sowie der Verformungen ist insbesondere darauf $\mathrm{zu}$ achten:

- Der erforderliche Wellendurchmesser darf nicht größer als der Fußkreisdurchmesser des Ritzels werden.

- Schwächungen des Durchmessers durch Einstiche für Wellensicherungsringe und Paßfedernuten sind durch Erhöhen des Durchmessers auszugleichen.

- Kerbwirkungen an Absätzen, Einstichen und Nuten müssen bei der Dimensionierung beachtet und bei der Gestaltung möglichst vermieden werden.

- Die Übertragung des Drehmomentes von der Welle zur Nabe erfordert möglicherweise einen größeren Wellendurchmesser.

- Bei Einsatz einer Keilwellenverbindung ist auf genormte Durchmesserreihen zu achten.

- Die Wahl eines Wälzlagers, das die geforderte Lebensdauer einhält, erfordert möglicherweise einen größeren Wellendurchmesser.

- Die seitliche Anlage des Wälzlagers (Mindestschulterhöhe nach Angabe des Herstellers) erfordert mögli- cherweise einen größeren Wellenabsatz.

- Normteile wie Sicherungsringe oder Dichtungen erfordern die Einhaltung von Vorzugsdurchmessern.

- Nach den erforderlichen Wellenabstufungen und Fügefasen muß der Durchmesser an der Kupplung noch ausreichend sein.

\subsubsection{Konstruktive Abhängigkeiten zwischen den Bauteilen}

Die konstruktive Realisierbarkeit ohne erheblichen Mehraufwand ist bei folgenden Entwurfsergebnissen zu überprüfen: Ist der Achsabstand ausreichend, um eine Flanschverschraubung zwischen den beiden Lageraußendurchmessern zu ermöglichen (s. Bild 2-5)? Ist das nicht der Fall, muß ein anderes Lager mit einem kleineren Außendurchmesser gewählt oder der Achsabstand vergrößert werden. Nach [1] ist für die betrachteten Baugrößen mindestens eine Schraube M12 erforderlich, der Abstand zwischen den Lageraußendurchmessern sollte mindesten $20 \mathrm{~mm}$ betragen.

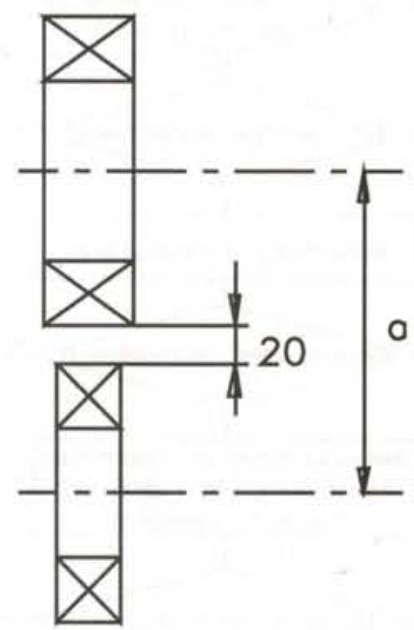

Bild 2-5: Platzbedarf für Verschraubung am Lagerflansch

Berührt oder überschneidet der Kopfkreisdurchmesser eines Zahnrades den Durchmesser der nächsten Welle (s. Bild 2-6)? Wenn der Wellendurchmesser nicht oder nur geringfügig verändert und das Zahnrad nicht verkleinert werden kann, ist der Achsabstand zu erhöhen.

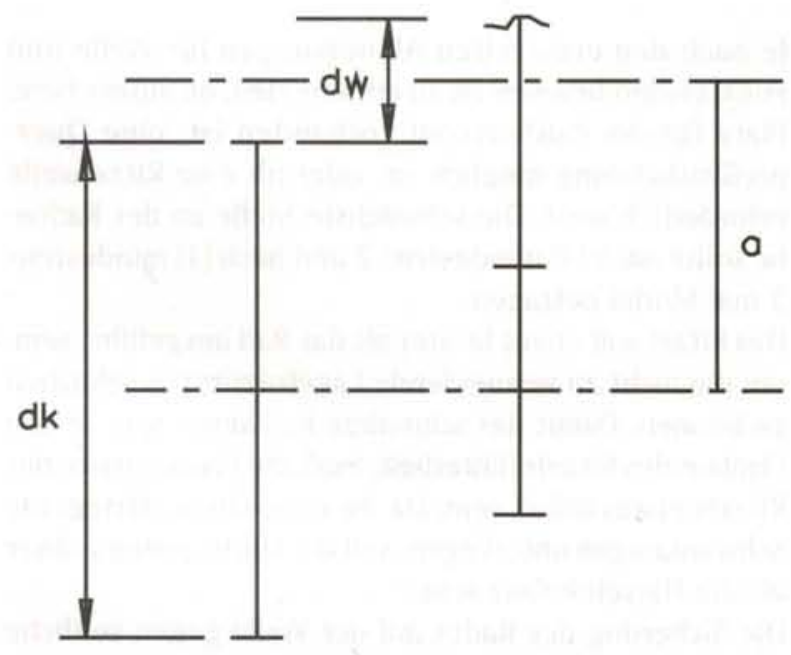

Bild 2-6: Platzverhältnisse zwischen Rad und Welle 


\subsubsection{Algorithmus für den Entwurf eines mehrstufigen Stirnradgetriebes}

Ausgehend von den Zusammenhängen der einzelnen Getriebebauteile hinsichtlich Berechnung und Gestaltung wurde folgender Berechnungsablauf entwickelt:

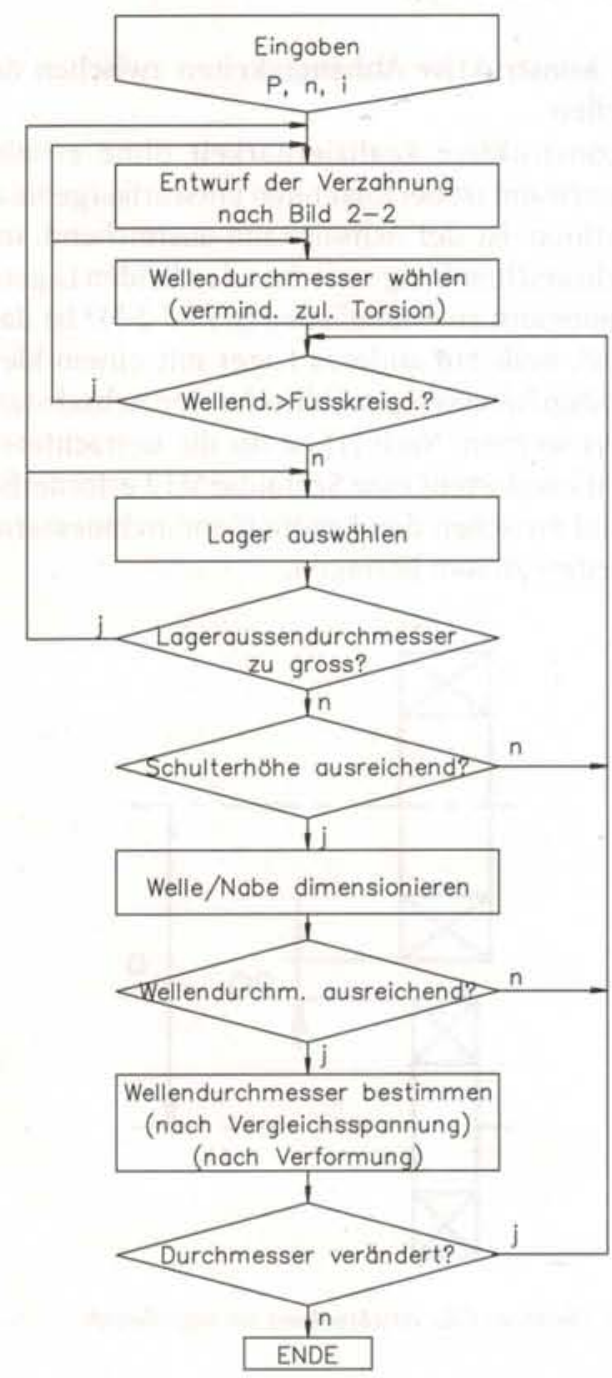

Bild 2-7: Schema zum Entwerfen von Stirnradgetrieben

\section{Gesichłspunkte für die konstruktive Gestaltung}

Je nach den ermittelten Abmessungen für Welle und Fußkreisdurchmesser ist zu entscheiden, ob ausreichend Platz für die Paßfedernut vorhanden ist, eine Querpreßverbindung möglich ist, oder ob eine Ritzelwelle erforderlich wird. Die schwächste Stelle an der Radnabe sollte nach $[4]$ mindestens 2 und nach $[1]$ mindestens 3 mal Modul betragen.

Das Ritzel soll etwas breiter als das Rad ausgefuihrt sein, um die nicht zu vermeidende Lagetoleranz ausgleichen zu können. Damit das schmalere Rad keine Spur in den Flanken des Ritzels hinterläßt, muß die Flankenhärte des Ritzels etwas höher sein. Da die errreichten Härtegrade Schwankungen unterliegen, soll der Härtesprung größer als die Härtetoleranz sein.

Die Sicherung des Rades auf der Welle gegen seitliche Verschiebung kann erfolgen durch:
- Reibschluß bei Querpreßverbindung,

- leichten Preßsitz bei geradverzahnten Rädern,

- seitliche Anlage an einem Wellenbund,

- seitliche Anlage an einem anderen Bauteil (Zahnrad, Abstandsring),

- Wellensicherungsring.

Ist die für das Wälzlager geforderte Mindestschulterhöhe nicht eingehalten, kann der Wellendurchmesser vergrößert oder ein Abstandring vorgesehen werden. Zur sicheren Anlage ist der Wellenabsatz etwas zurückzunehmen (s. Bild 3-1).

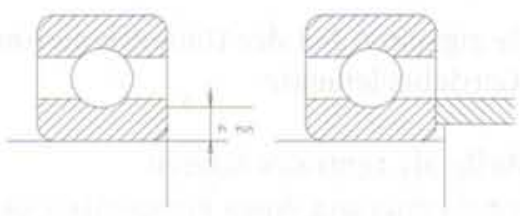

Bild 3-1: Gewährleistung der Mindestschulterhöhe am Lager

Werden die Radnaben seitlich abgesetzt, lassen sich die Räder platzsparend ineinanderschachteln (s. Bild 3-2). Bei dieser Anordnung greifen die Zahnkräfte weiter am Lager an, was eine geringere Biegespannung und Verformung der Welle bewirkt.

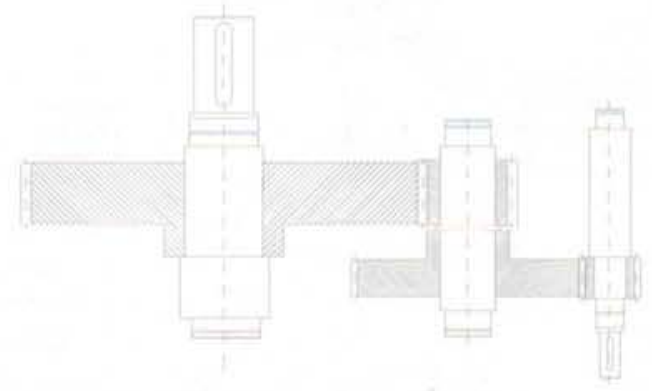

Bild 3-2: Anordnung der Räder

Die Anordnung des Festlagers kann nach folgenden Gesichtspunkten festgelegt werden:

- Das Festlager auf der Kupplungsseite vermindert Längsverschiebungen an der Kupplung.

- Festlager wird das Lager, welches die geringere Radialkomponente aufnehmen muß, damit wird eine gleichmäßigere Auslastung beider Lager einer Welle gewährleistet.

- Das Festlager wird so angeordnet, daß die Axialkraft auf kurzem Weg zum Lager geleitet wird.

Stützlagerungen sind die einfachste Lagerkonstruktion, allerdings müssen beide Lager die Axialkraft aufnehmen können.

Für die Auswahl und den Einsatz der verschiedenen Lagerbauarten sind die Hinweise und Empfehlungen der Hersteller zu beachten.

Bei der Wellengestaltung ist auf Folgendes zu achten:

- Für die leichtere Montage der Bauteile sind an der Welle verschiedene Fügefasen vorzusehen.

- Die Wellenabsätze sollen keine Stellen mit hohen Kerbwirkungen haben. Einstiche und Nuten sind zu vermeiden bzw. an Stellen mit geringer Beanspruchung anzuordnen. 


\section{Programmierung für eine durch- gängige Getriebebearbeitung am PC}

\subsection{Wahl des CAD-Systems}

Für die grafische Arbeit am Bildschirm stehen an der Technischen Fachhochschule Wildau folgende Programme zur Verfügung:

CAD-Labor im FB Maschinenbau: AutoCAD GENIUS

CAM-Labor im FB Maschinenbau: SuperDRAFT Hochschulrechenzentrum: AutoCAD unter AIX-WINDOWS Pro/ENGINEER CADDY

Da das Programm WCAD hauptsächlich im Fach Konstruktion Anwendung finden soll, bietet sich dafür das CAD-Labor mit den Arbeitsplätzen für ACAD und GENIUS an. ACAD und Genius sind weit verbreitete CAD-Programme, für die bereits zahlreiche Applikationen zur Verfügung stehen. Die Weiterbearbeitung der Entwurfsgrafik stellt für den Studenten kein Problem dar, da diese Programme bereits im Grundstudium zur Anwendung kommen. Über entsprechende Schnittstellen (z. B. DXF) ist die Grafik auch an andere Systeme transferierbar.

\subsection{Wahl des Nachrechnungsprogrammes}

Anforderungen an die Nachrechnungsprogramme:

- Verzahnungsgeometrie,

- Festigkeitsnachweis für Zahnräder,

- Berechnung von Wellen auf Dauerfestigkeit und Verformung,

- Lebensdauer der Wälzlager,

- Paßfederverbindungen, Keilwellen und Querpreßverbindungen.

- Die Nachrechnungsprogramme müssen auf PCs der verschiedenen Generationen laufen. Die Speichermöglichkeit für Daten in Netzen ist erwünscht.

- Die Nachrechnung ist durch übersichtliche Protokolldrucke ausreichend zu dokumentieren.

- Der Dialog sollte auf die Besonderheiten der Lehre zugeschnitten sein und insbesondere Hilfe bei der Bestimmung von Werten leisten.

- Die einzelnen Programme sollten unter einem einheitlichen Dialog abgearbeitet werden können, um notwendige Einarbeitungszeiten zu begrenzen.

- Eine laufende Aktualisierung der Nachrechnungsprogramme sollte gewährleistet sein.

- Eine Einbindung in den Rahmen von WCAD ist erforderlich, damit die Entwurfsdaten ohne zeitaufwendige und möglicherweise fehlerbehaftete Neueingabe in die Nachrechnung übergeben werden können. Dazu muß die Dateistruktur des Nachrechnungsprogrammes zugänglich sein.

Von den verschiedenen, zum Teil auch von Studenten programmierten Nachrechnungsprogrammen, die an der TFH Wildau verfügbar sind, erfüllt das Programm ME DESIGN von der Firma TEDATA diese Anforderungen.

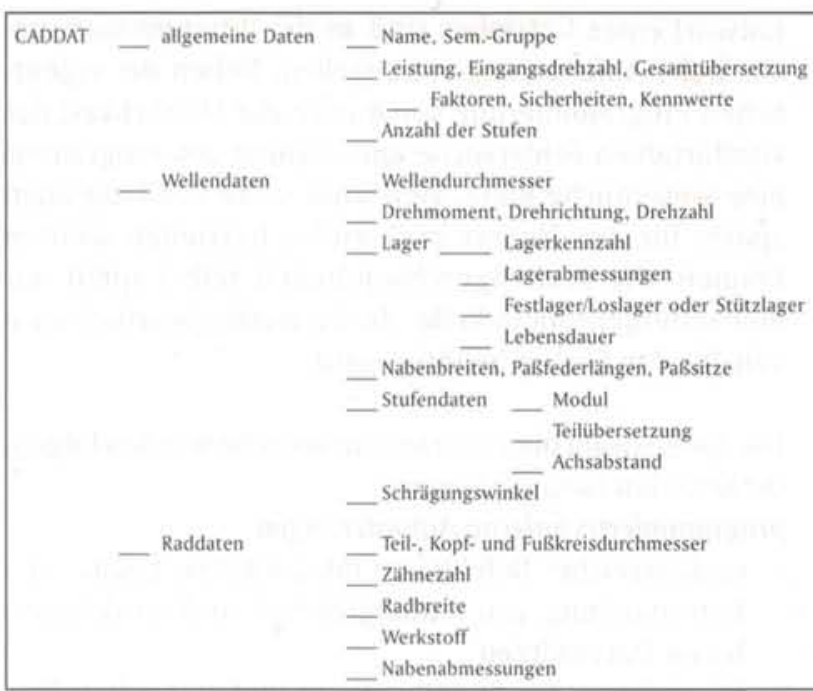

Bild 4-1: Struktur des WCAD-Datensatzes

\subsection{Dateien, Datenformate und Schnittstellen}

Alle veränderlichen Daten wurden zu dem Datensatz CADDAT zusammengefaßt. Dieser kann mit Borland Pascal 7.0 problemlos gespeichert und geladen werden. Die Variablen des Datensatzes sind folgendermaßen strukturiert: Neben der aktuellen Projektdatei CADDAT, in der aktuelle Eingaben und Ergebnisse abgespeichert sind, existieren

- dem Nutzer zugängliche Dateien:

Werkstoffe

Lager

Hilfedatei

Konfigurationsdatei

- programminterne Dateien:
Paßfedern
Preßpassungen
Keilwellenprofile
Vorzugsreihen für Modul, Achsabstände Teilïbersetzungen
Einstich für Wellensicherungsringe Wellenenden

Für die Übergabe der berechneten Daten an ein Grafikprogramm müssen die Daten so aufgearbeitet werden, daß sie dem Datenformat der Grafikprogramme entsprechen. Hierzu bietet sich die Nutzung der meist zu den Grafikprogrammen mit angebotenen Programmiersprachen an. Für AutoCAD ist das z. B. die Programmiersprache AutoLISP. Das Schreiben der Daten direkt in eine DATEI.DWG entsprechend dem Zeichnungsformat bei AutoCAD oder in eine neutrale Schnittstelle z. B. DATEI.DXF ist theoretisch ebenfalls möglich, praktisch aber nur sehr schwer handhabbar. Nachteilig bei Nutzung der auf AutoCAD zugeschnittenen Programmiersprache wirkt sich jedoch aus, daß die Grafikübergabe nur an AutoCAD oder über einen Konverter, der DWGDateien in neutrale Formate umwandelt, erfolgen kann.

\subsection{Auswahl der Programmiersprache}

Für die Programmierung einer solch umfangreichen und komplexen Konstruktionsaufgabe wie dem kompletten 
Entwurf eines Getriebes sind an die Programmiersprache hohe Anforderungen zu stellen. Neben der eigentlichen Programmierung spielt auch die Möglichkeit der komfortablen Fehlersuche und Testung des Programms eine wesentliche Rolle. Weiterhin sollte das Programm später für den Nutzer problemlos betrieben werden können. Die Rechengeschwindigkeit selbst spielt nur eine untergeordnete Rolle, da die meiste Bearbeitungszeit für den Dialog benötigt wird.

Für die Auswahl der Programmiersprache wurden folgende Kriterien herangezogen:

\section{programmiertechnische Anforderungen}

- umfangreicher Befehlssatz (Mathematik, Logik, ...)

- Bereitstellung von umfangreichen und strukturierbaren Datensätzen

- Möglichkeit der Erstellung von umfangreichen Programmen und deren Strukturierung

- schnelle Compilierung des Quelltextes bei der Programmierung und Testung neuer Programmabschnitte

- Möglichkeiten der Fehlersuche (Fehlererkennung im Syntax, Debugger u. a.)

- Vermeidung von Programmabstiurzen

- direkte Ansteuerung von Bildschirm, Drucker und Disk

Anforderungen aus der verfügbaren Hardware

- Programmierung für PC-Generation

benutzerfreundliche Anforderungen

- lauffähiges compiliertes Programm

- Rechengeschwindigkeit ist ohne besondere Bedeutung

Die Ergebnisse des Vergleiches verschiedener geläufiger Programmiersprachen sind in Tab. 4-1 zusammengefaßt. Dabei wurden nur die Sprachen selbst, aber nicht spezielle Compiler oder Interpreter berücksichtigt.

\begin{tabular}{|c|c|c|c|c|c|c|c|}
\hline \multirow[t]{2}{*}{ Kriterien } & \multicolumn{3}{|c|}{ Hochsprachen } & \multicolumn{2}{|c|}{ einf. Sprachen } & \multicolumn{2}{|c|}{ Logiksprachen } \\
\hline & c & Modula & Pascal & Basic & Clipper & LISP & Prolog \\
\hline Befehlssatz & ++ & ++ & ++ & + & + & 0 & 0 \\
\hline Datensatz & ++ & ++ & ++ & 0 & 0 & + & - \\
\hline Strukturierung & ++ & ++ & ++ & - & - & - & -- \\
\hline Programmsprünge & 0 & 0 & + & ++ & ++ & -- & -- \\
\hline compiliert lauffähig & ++ & ++ & ++ & - & 0 & -- & - \\
\hline Fehlersuche & + & + & ++ & 0 & - & 0 & 0 \\
\hline Programmtest & ++ & ++ & ++ & + & + & + & 0 \\
\hline Länge Quelltext & ++ & ++ & ++ & + & + & 0 & -- \\
\hline Programmlänge & ++ & ++ & ++ & 0 & - & - & - \\
\hline Gesamtneigung & ++ & ++ & ++ & + & + & - & - \\
\hline
\end{tabular}

Tab. 4-1: Gegenüberstellung der Programmiersprachen

Es wird deutlich, daß mit Borland Pascal 7.0 eine leistungsfähige Programmiersprache zur Verfuigung steht, die die notwendigen Anforderungen besonders gut erfüllt. Einige interne Probleme von Borland Pascal bei der Verwaltung von großen Datenmengen machten eine teilweise umständliche Programmierung nötig.

\subsection{Programmstruktur}

Das Programm ist in einzelne Module aufgeteilt. Dazu wird das UNIT-Konzept von Turbo-Pascal benutzt. Um
Speicher zu sparen, wird der Overlay-Manager verwendet.

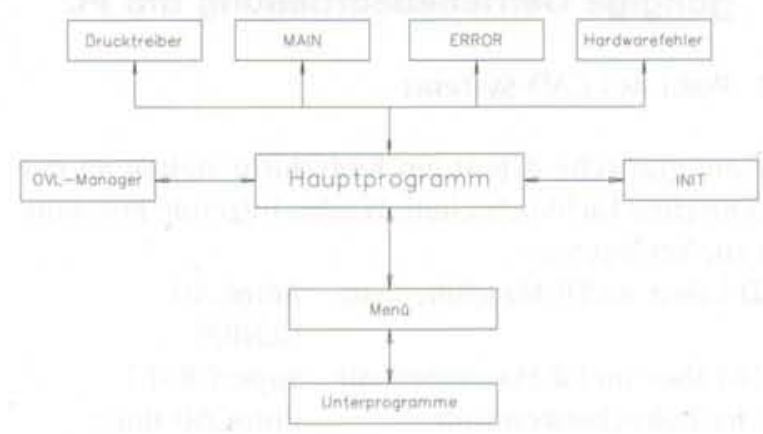

Bild 4-2: Programmstruktur WCAD

Die einzelnen Grundmodule haben dabei folgende Bedeutung:

Hauptprogramm: übernimmt Parameter vom Start, lädt andere Programmteile nach, startet Menuiführung

Druckertreiber: stellt die Verbindung zum gewünschten Drucker her

Main: $\quad$ Ein-/Ausgabe auf/von Diskette, Konsole und Drucker, Ansteuerung EGAVGA, Daten: Werkstoffdatei, Lager, Entwurfswerte, Variablen

Error: $\quad$ Fehlerabfangroutine fuihrt zum definierten Abbruch bei Fehlermeldung, speichert alle berechneten Daten

Hardwarefehler: reagiert bei Disk- oder Druckerfehlern mit Abbrechen, Wiederholen, $\underline{\text { Überge- }}$ hen oder Beenden

Init: lädt Werkstoff- und Lagerdateien, initialisiert Grafikkarte, lädt die Standardwerte

OVL-Manager: organisiert, daß nur die nötigen Programmteile im Speicher sind

Menü: $\quad$ ruft nach Auswahl den entsprechenden Programmteil auf U-Programme Entwurfsberechnungen und Grafik

\subsection{Grafikschnittstelle}

Für eine Übergabe der Berechnungsdaten an AutoCAD und eine graphische Darstellung der berechneten Bauteile sind folgende Schritte erforderlich: Die Welle wird in verschiedene Formelemente wie Viereck, Linie, Fase, Langloch und Text aufgeteilt und fortlaufend numeriert (s. Bild 4-4). Dabei kann sich ein Formelement, wie z. B. der Nabensitz von Rad 2, je nach konstruktiver Ausführung in der Lage und Form verändern. Die Koordinatendaten für diese Formelemente werden nach ergänzenden Abfragen aus den Entwurfsdaten berechnet. Diese Elemente werden an die Unit interface übergeben und in eine AutoLISP-Datei geschrieben.

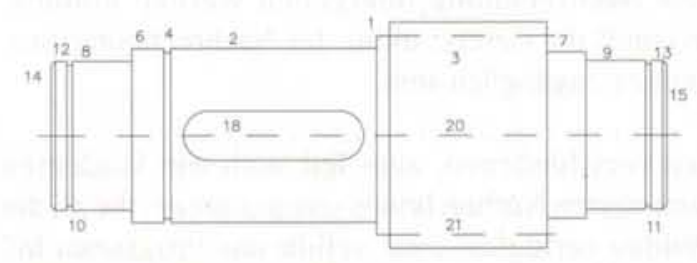

Bild 4-4: Definition der Formelemente 
Die einzelnen Formelemente sind z. B. wie folgt zugeordnet:

\begin{tabular}{|c|c|c|}
\hline 1 & Bund & Viereck \\
\hline 2 & Sitz des Zahnrades & Viereck \\
\hline 3 & Sitz des Ritzels & Viereck \\
\hline $4,5,11,13$ & $\begin{array}{l}\text { Einstich für Wellen- } \\
\text { sicherungsring }\end{array}$ & Viereck \\
\hline $7,7,12,13$ & konstruktive Abstände & Viereck \\
\hline 14,15 & Fase & Fase \\
\hline 18,19 & Paßfedernut & Langloch \\
\hline 20 & Mittellinie der Welle & Linie \\
\hline 21 & Teilkreislinie & Linie \\
\hline ab 30 & Texteintragungen & Text \\
\hline
\end{tabular}

Für die verschiedenen möglichen Gestaltvarianten mußte von folgenden Fällen ausgegangen werden:

Antriebswelle und Abtriebswelle:

1. Kupplung re., Bund re., Ritzel re. 4. Spiegelfall von 1.

2. Kupplung lks., Bund re,, Ritzel re. 5. Spiegelfall von 2.

3. Kupplung lks., Bund lks., Ritzel re. 6. Spiegelfall von 3.

Um die Anzahl der möglichen Varianten nicht zu groß werden zu lassen, wird der Bund der Zwischenwelle nur mittig angeordnet. Soll die Montage beider Räder von der gleichen Seite erfolgen, kann der Bund nachträglich im CAD-System editiert werden. Je nach Anordnung von Rad und Ritzel ist die Spiegelung möglich.

Für den größten Wellendurchmesser am Bund wird ein Wert aus der Halbzeugtabelle für Rundmaterial ausgewählt. Dieser Durchmesser erscheint auch im Schriftfeld bei der Bezeichnung von Halbzeug/Werkstoff.

Räder werden in Halbschnitt/Halbansicht (s. Bild 4-5) dargestellt. Damit kann durch Löschen und Spiegeln bzw. Wahl der entsprechenden Layer das Rad in der Ansicht oder als Schnitt generiert werden. Auch hier wurden Formelemente vereinbart, die vor allem aus Linien und Text bestehen. Um die verschiedenen Radformen gestalten zu können und die Schraffur in ACAD zu gewährleisten, wird das Rad aus Teillinienstücken zusammengesetzt.

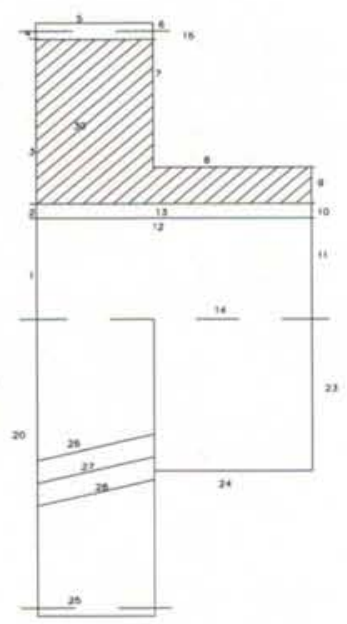

Bild 4-5: Definition der Formelemente am Zahnrad

Für diese Formelemente werden mit den entsprechenden Entwurfsdaten die erforderlichen Koordinaten berechnet. Für jedes dieser Formelemente existiert ein Un- terprogramm, mit dem diese Daten in ein AutoLISP-Programm übersetzt und gespeichert werden. Je nach Gestaltungsvariante können dabei Spiegelungen erforderlich werden. Die gesamte Zeichnung wird danach auf dem Zeichenblatt zentriert.

Damit die Grafikdaten von AutoCAD aus aufgerufen werden können, mußte die AutoCAD-Datei ACAD.LSP geändert werden. Dadurch wird die LISP-Prozedur (WCAD) erzeugt. Diese lädt das erforderliche Formblatt mit den Standardeinstellungen, stellt die notwendigen Dialogabfragen und öffnet die entsprechende LISP-Datei. In dieser befinden sich die eigentlichen Zeichenbefehle. Das Formblatt mit Zeichenblatt, Schriftfeld, Verzahnungstabelle, vorbereiteten Layern und Grundeinstellungen ist als DXF-Datei angelegt. Es stehen die Formate DIN A3 und DIN A2 mit oder ohne Verzahnungstabelle zur Verfügung. Die Zeichnungen werden grundsätzlich im Maßstab 1:1 angezeigt. Eintragungen im Schriftfeld und der Verzahnungstabelle werden so weit wie vorhanden eingetragen. Deshalb ist es ratsam, die Grafik der Zahnräder erst nach der Erstellung der Verzahnungsgeometrie aufzurufen.

Die Bilder 4-6 und 4-7 zeigen Beispiele für die WCADGrafik einer Welle und eines Zahnrades. Die Weiterbearbeitung zur bemaßten Einzelteilzeichnung oder zur Baugruppenzeichnung erfolgt mit dem CAD-System.

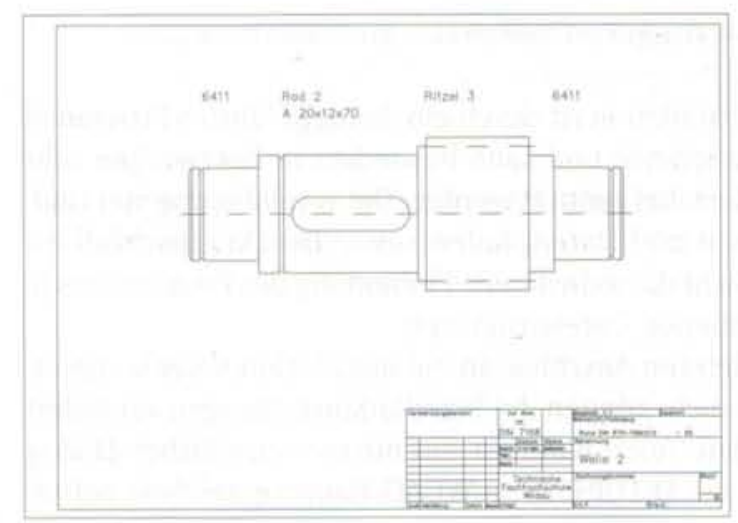

Bild 4-6: WCAD-Zeichnung Welle

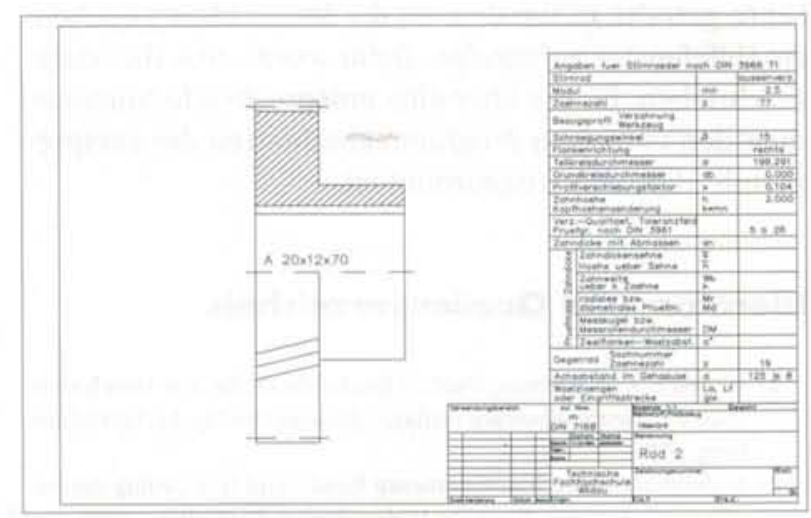

Bild 47: WCAD-Zeichnung Zahnrad

\subsection{Programmtechnische Besonderheiten}

Bei der Programmierung wurde besonderer Wert auf die Nutzerfreundlichkeit gelegt. Der Bildschirm ist farbig gestaltet und übersichtlich eingeteilt. Im Kopf ist der 
Lizenznehmer und der Programmname eingetragen. Die Fußzeile zeigt an, in welchem Menüpunkt man sich befindet.

Besonders eindeutig wird der Dialog durch zusätzliche Zeichen, die im ASCII-Format entwickelt wurden. Dazu ist eine Matrix von $16 \times 16$ Quadraten definiert, und je nach gewünschtem Zeichen sind die einzelnen Felder geschwärzt oder frei. Für jeweils $4 \times 4$ Spalten sind die Codezahlen 8, 4, 2 und 1 zugeordnet. Für jede Zeile wird die Summe der geschwärzten Felder als 4-stelliger Code dargestellt, wobei die Summen im hexadezimalen Zahlensystem dargestellt und von 10 bis 15 mit den Buchstaben A bis F belegt sind. Bild 4-9 zeigt ein Beispiel. Es sind insgesamt 21 Sonderzeichen codiert. Damit die Anzeige am Bildschirm richtig erfolgt, muß der deutsche Zeichensatz (Code 437) eingestellt sein.

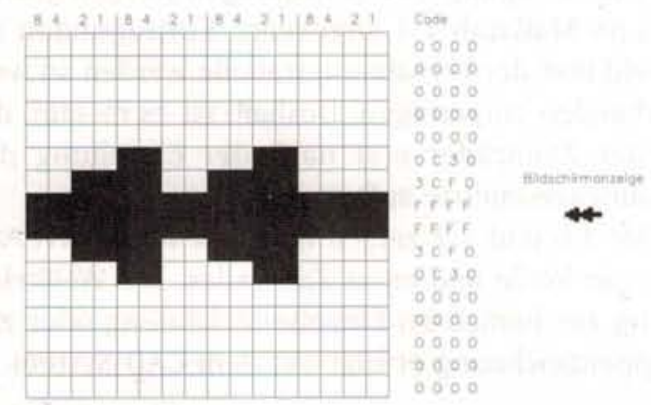

Bild 49: Erzeugen von Sonderzeichen für die Bildschirmanzeige

Die Installation ist durch ein dialoggeführtes Programm vorzunehmen und kann besonders in Netzwerken sehr komfortabel genutzt werden. Die Vereinbarung von Laufwerken und Datenpfaden sowie Druckeranschluß ermöglicht die individuelle Einbindung des Programmes in bestehende Dateistrukturen.

Im direkten Anschluß an die Installation, oder wenn erforderlich, können die Standardeinstellungen verändert werden. Auch dafür ist ein nutzerfreundlicher Dialog über die SETUP.EXE im WCAD-Hauptverzeichnis aufrufbar.

Um den besonderen Anforderungen des Einsatzes in der Lehre gerecht zu werden, ist die Möglichkeit gegeben, ein Hilfefenster aufzurufen. Dafür wurde eine Hilfedatei geschrieben, für die über eine entsprechende Numerierung den einzelnen Programmabschnitten die entsprechende Hilfeseite zugeordnet ist.

\section{Literatur- und Quellenverzeichnis}

[1] W. Beitz, K.-H. Küttner, Dubbel Taschenbuch für den Maschinenbau, 17., neubearbeitete Auflage, Springer-Verlag Berlin/Heidelberg 1990

[2] G. Niemann, Maschinenelemente Band I und II, 2., völlig neubearbeitete Auflage, Springer-Verlag Berlin/ Heidelberg 1989

[3] R. Hinz. Verbindungselemente Achsen Wellen Lager Kupplungen, 1. Auflage, Fachbuchverlag Leipzig 1984

[4] K. Zirpke, Zahnräder, 13. Auflage, Fachbuchverlag Leipzig 1989

[5] K. Wächter, Konstruktionslehre für Maschineningenieure, 1. Auflage, Verlag Technik Berlin 1987

[6] Roloff/Matek, Maschinenelemente, 12., neubearbeitete Auflage, Vieweg-Verlag Braunschweig/Wiesbaden 1992

[7] Decker, Maschinenelemente, 10 ., vollständig überarbeitete und erw. Auflage, Carl Hanser Verlag München 1990
[8] Köhler/Rögnitz, Maschienenteile, 8 , neubearbeitete und erweiterte Auflage, B. G. Teubner Stuttgart 1992

[9] Tischer, PC intern, 1. Auflage, DATA BECKER GmbH Düsseldorf 1992

[10] M. Althaus, Turbo Pascal Profibuch, 2. Auflage, SYBEX-Verlag GmbH Düsseldorf 199

[11] AUTOCAD Handbuch, Autodesk AG Schweiz

[12] AutoLISP. Handbuch für den Programmierer, Autodesk AG Schweiz

[13] Born, Referenzhandbuch Dateiformate, 1. Auflage 1991, Addison-Wesley Publishing Company

[14] ME DESIGN 5.0, Dokumentation I, II und III, 4.. ergänzte Auflage, TEDATA GmbH Bochum 1993

[15] H.-1. Paschiller, Ingenieurabschlußarbeit, Ingenieurschule für Maschinenbau Wildau 1993

[16] FAG Wälzlager Standardprogramm. Ausgabe März 1991, FAG Kugelfischer Georg Schäfer KGaA Schweinfurt

[17] L. Horn, Ingenieurabschlußarbeit, Ingenieurschule für Maschinenbau Wildau 1993

\section{Verfasser}

Prof. Dipl.-Ing. Karin Jannasch

Technische Fachhochschule Wildau

Fachbereich Maschinenbau

Tel. (03375) 507-161 